\title{
High-contrast Background-free Magnetic Resonance Molecular Imaging
}

\author{
Sayoni Ray ${ }^{1 *}$, Chao-Hsiung Hsu ${ }^{1,2}$, Zhao Li ${ }^{1}$, Fang-Chu Lin ${ }^{1}$, Yung-Ya Lin ${ }^{1}$ \\ ${ }^{1}$ Department of Chemistry and Biochemistry, University Of California, Los Angeles, USA \\ ${ }^{2}$ Department of Chemistry, National Taiwan University, Taiwan \\ *Corresponding author: sayoniray@ucla.edu
}

Number of references: 48

Number of figures and tables: 8 figures

Supplementary Material, 1 figure 


\begin{abstract}
$\underline{\text { Abstract }}$
One of the holy grails in cancer detection and therapy is to simultaneously image and deliver drugs to the tumor site using a safe nanoparticle. Liposomes are a well-known safe and stable nanoparticles that can be loaded with a drug and suitable contrast agent for magnetic resonance imaging (MRI). However, the loading of a contrast agent such as gadolinium in liposomes generally results in poor contrast and various artifacts in in vivo experiments, compared to free gadolinium chelate experiments. Current work demonstrates the effective filtering of artifacts and contrast enhancement to obtain high quality images of the tumor sites in mouse models using paramagnetic liposome nanoparticles as contrast agent, a novel pulse sequence in active-feedback MRI, and nonlinear fitting. Our results show a significant improvement in eliminating artifacts and increasing contrast compared to the standard MRI techniques, and a close correlation with histopathology and inductively coupled mass spectrometry results. This newly developed protocol could be used for any paramagnetic nanoparticle to improve detection sensitivity. We expect this methodology to produce similar improvements in human imaging, having the potential to significantly improve early tumor detection in clinical practice and opening up the possibility of its theranostic use.
\end{abstract}

Keywords: paramagnetic liposome, gadolinium (Gd), active-feedback magnetic resonance imaging (MRI), difference imaging, high-contrast, sensitive imaging, background free, artifactfree 


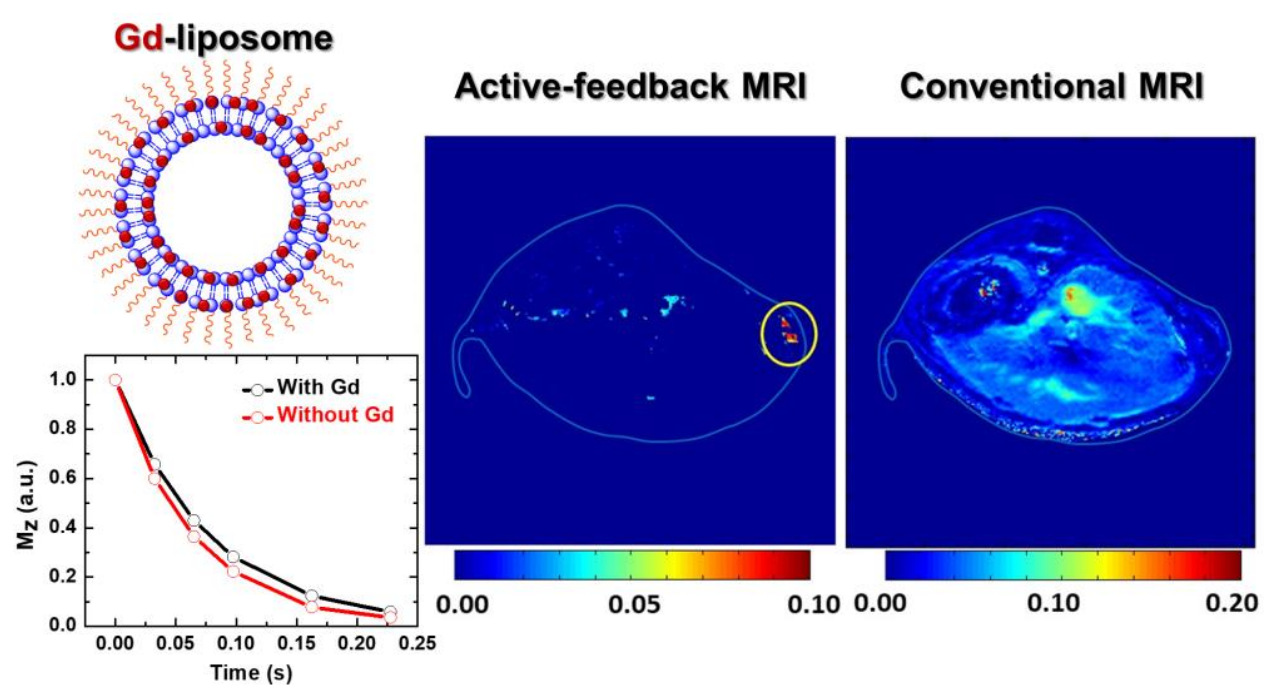

\section{Graphical Abstract}

\section{Introduction}

Magnetic resonance imaging (MRI) is a non-invasive tool to obtain high-resolution anatomical images of a tumor site. Recent advances ${ }^{1-4}$ in targeting a tumor site by nanoparticles such as liposomes ${ }^{5-7}$ carrying both drug and an MRI contrast agent have added a new dimension in cancer therapy. The possibility of online monitoring of the tumor site during the drug release process, as well as the detection of early tumors, would significantly improve the prognosis of cancer patients. However, MR imaging suffers from poor contrast and the problem of artifacts that originate from calcification, fat, hemorrhage, blood clots etc., mimicking MRI signals from the contrast agent. ${ }^{8}$ The problem is further aggravated when the imaging is performed by loading the contrast agent in a nanoparticle such as a liposome for theranostic applications.

Biocompatibility, size controllability, and functional versatility make liposomes ideal nanovehicles for carrying and delivering cargo inside the human body. Such self-assembled spherical lipid bilayer are already clinically approved ${ }^{5,6,9-12}$ and are capable of carrying both 
hydrophilic and hydrophobic drugs in their aqueous core and in lipid membrane respectively. Liposomal nanoparticles can be modified into in vivo long-circulating nanovehicles by engineering them with a conjugating moiety and polyethylene glycol (PEG) for active and passive targeting, respectively. $\mathrm{Gd}^{3+}$ chelates have been extensively studied in the past and a couple of them have been approved as MRI contrast agents for clinical use. However, for theranostic use, $\mathrm{Gd}^{3+}$ chelates have to be encapsulated in a nanoparticle such as a liposome that can simultaneously carry drug molecules and target the tumor site. Moreover, the toxicity of $\mathrm{Gd}^{3+}$ for renal patients could be somewhat alleviated by its encapsulation in liposomes. On the other hand, the encapsulation of $\mathrm{Gd}^{3+}$ restricts the relaxivity of the contrast agent compared to the free chelate due to limited water flux between the aqueous core and the outside bulk water, ${ }^{13-16}$ resulting in limited contrast and the problem of artifacts in the conventional $\mathrm{T}_{1}$-weighted images. There have been many efforts to solve these problems in previous studies, ${ }^{13,16-18}$ such as optimizing the size of the liposomes, increasing the load of $\mathrm{Gd}^{3+}$ - chelates, etc., however the problem still exists.

Contrast in conventional MRI techniques largely depends on the differing molecular dynamics of different tissues, resulting in variations of the relaxation times of the signal. ${ }^{19}$ These imaging techniques have limited efficiency to differentiate between tissues or materials with only a slight difference in the relaxation parameters. The online monitoring of the tumor or the detection of the early tumors is associated with a slight shift in magnetic susceptibility and conventional MR imaging techniques often fail to identify such small changes. This meager shift in the local field is independent of molecular dynamics and is thus essentially invisible to the conventional relaxation-based imaging mechanisms such as $\mathrm{T}_{1}, \mathrm{~T}_{2}$ or $\mathrm{T}_{2}{ }^{*}{ }^{19,20}$ Another related methodology, susceptibility-weighted imaging $(\mathrm{SWI})^{21}$ is a post-processing technique where further enhancement of the contrast is achieved by applying phase mapping to the $\mathrm{T}_{2} *$ images. 
However, SWI struggles in cases where $\mathrm{T}_{2}{ }^{*}$ decays limit the amount of phase shift, or where the resonance offset change across the imaging plane is inadequate. Hence, a highly sensitive and safer MRI approach capable of theranostic application is highly desirable for the online monitoring of the tumor during the drug release process at the tumor site and also for detecting early tumors.

It was demonstrated previously that the use of the radiation damping feedback field, a nonlinear approach that relied on Lenz's law, could enhance the contrast by amplifying a small magnetic difference of the system. ${ }^{22-26}$ In general, radiation damping effects are more pronounced in a high-field condition, but an active-feedback circuit can produce the same effect in a low magnetic field. A small change in the magnetization is collected and fed back into the sample in a loop during the evolution of the magnetization. Such nonlinear dynamics are highly sensitive to the initial magnetization conditions, and in some cases produce chaotic dynamics. ${ }^{23}$ The selective nature of this active-feedback field has been found to magnify the contrast between tissues with only a slight variation in magnetic properties. ${ }^{24-28}$ However, the reactionary nature of the previously used active-feedback ${ }^{27,29-36}$ pulse sequence efficiently return the sample magnetization back to its equilibrium orientation on a short time scale and limits the extent of the contrast by restricting its nonlinear evolution.

In this work, we have designed a new pulse sequence by introducing a continuous-wave (CW) radio-frequency field in the presence of an active-feedback field to extend the nonlinear evolution of magnetization over time. This improved pulse sequence having a CW component can stabilize and preserve the contrast against decay, and can ameliorate the sensitivity of the activefeedback method. We find from theoretical simulations of the active-feedback process that the new pulse sequence produces a characteristic biexponential temporal variation of the longitudinal magnetization in the difference images between the proton spins with and without Gd-liposomes. 
We have used the difference imaging technique with and without Gd-liposome (paramagnetic liposome) to locate a tumor site and have taken anatomical MR images of paramagnetic liposomes attached to the tumor site. Removal of artifacts and enhancement of contrast have been demonstrated by exploiting the biexponential temporal characteristic of MRI signal coupled with difference imaging. This new technique results in significantly superior imaging contrast of the positions of Gd-liposomes, compared to conventional MRI methods in subcutaneous glioblastoma multiform (GBM) mice models. These findings are verified by inductively coupled plasma mass spectrometry (ICP-MS) results.

\section{Results and Discussion}

\subsection{Material Characterization}

The liposomes were prepared by the thin layer evaporation method and Gd was incorporated into the bilayer. PEG lipids were incorporated into the surface of the liposome for longer circulation time in the blood in order to increase the chances of targeting cancer cells through the enhanced permeability retention (EPR) effect. Liposomes were characterized by negative stain transmission electron microscopy (TEM) and dynamic light spectroscopy (DLS). DLS study has shown that the nanoparticles have a mean diameter of $168.0 \mathrm{~nm}$ with standard deviation of $76.6 \mathrm{~nm}$, consistent with Figure 1 demonstrating the representative TEM images for the synthesized nanoparticles, indicating a uniform distribution and successful formation of bilayered liposomes. 

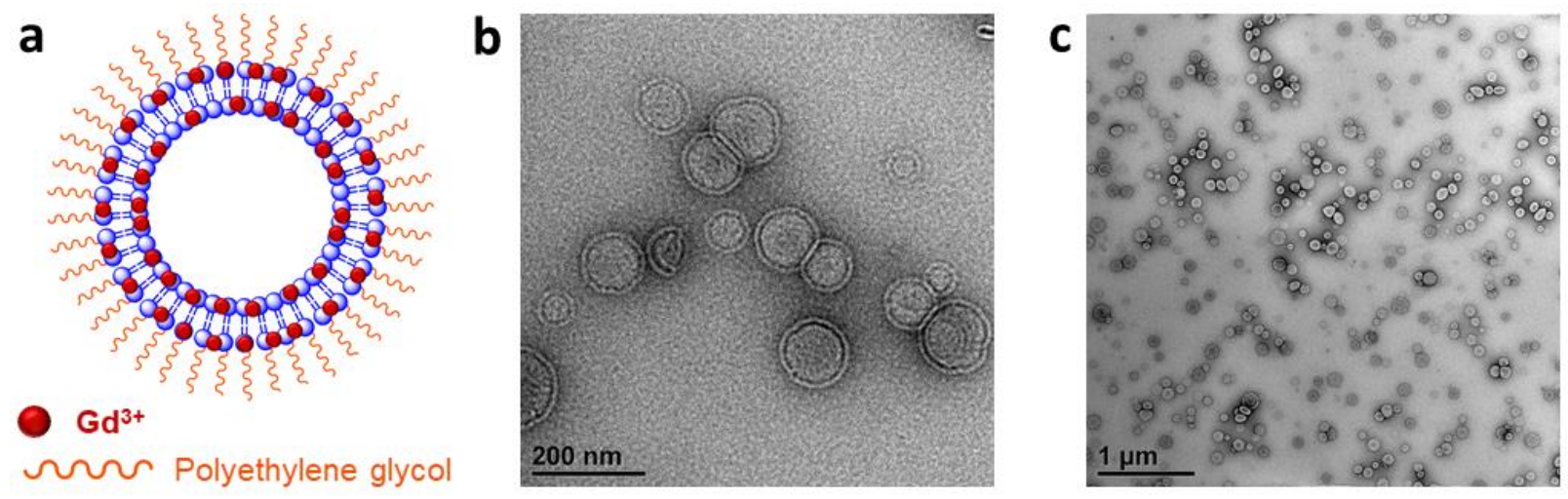

Figure 1. Schematic diagram with material characterization a) Schematic diagram of paramagnetic liposomes (Gd-liposomes), where red dots show the presence of Gd on the bilayer and the orange lines representing polyethylene glycol (PEG) for EPR effect b) TEM images show successful formation of bilayered liposome c) Demonstrates homogeneous distribution and morphology of nanoparticles under TEM.

\subsection{Relaxation Measurements}

Different relaxation parameters like longitudinal and transverse relaxation decay rates $\left(\mathrm{T}_{1}\right.$ and $\mathrm{T}_{2}$ ) at different concentrations were probed for the Gd-liposome solution in a $300 \mathrm{MHz}$ Varian microimaging setup and plotted to obtain the longitudinal and transverse relaxivity values, $\mathrm{r}_{1}$ and $\mathrm{r}_{2}$, respectively . Further, those were compared with the standard contrast agents, such as Gadavist and Magnevist. As shown in Figure 2, the nanoparticle solution exhibited an $r_{1}$ value of $1.68 \mathrm{~s}^{-1}$ $\mathrm{mM}^{-1}$, whereas Gadavist and Magnevist showed $\mathrm{r}_{1}$ values of 4.70 and $4.32 \mathrm{~s}^{-1} \mathrm{mM}^{-1}$ respectively. In contrast, the nanoparticles demonstrated a higher $\mathrm{r}_{2}$ value of $8.93 \mathrm{~s}^{-1} \mathrm{mM}^{-1}$ compared to for Gadavist and $5.34 \mathrm{~s}^{-1} \mathrm{mM}^{-1}$ for Magnevist. Hence, the prepared Gd-liposomes are much weaker $\mathrm{T}_{1}$ contrast agents, compared to the standard, clinically used Gd compounds, whereas they are much stronger $T_{2}$ contrast agents compared to others. This feature of Gd-nanoparticles could be attributed to their particular structure, where the amphiphilic Gd-DTPA-BSA molecules were incorporated into the hydrophobic bilayer of the liposome through the hydrophobic interaction. The hydrophilic head with $\mathrm{Gd}^{3+}$ should orient itself towards either the inner aqueous core of the 

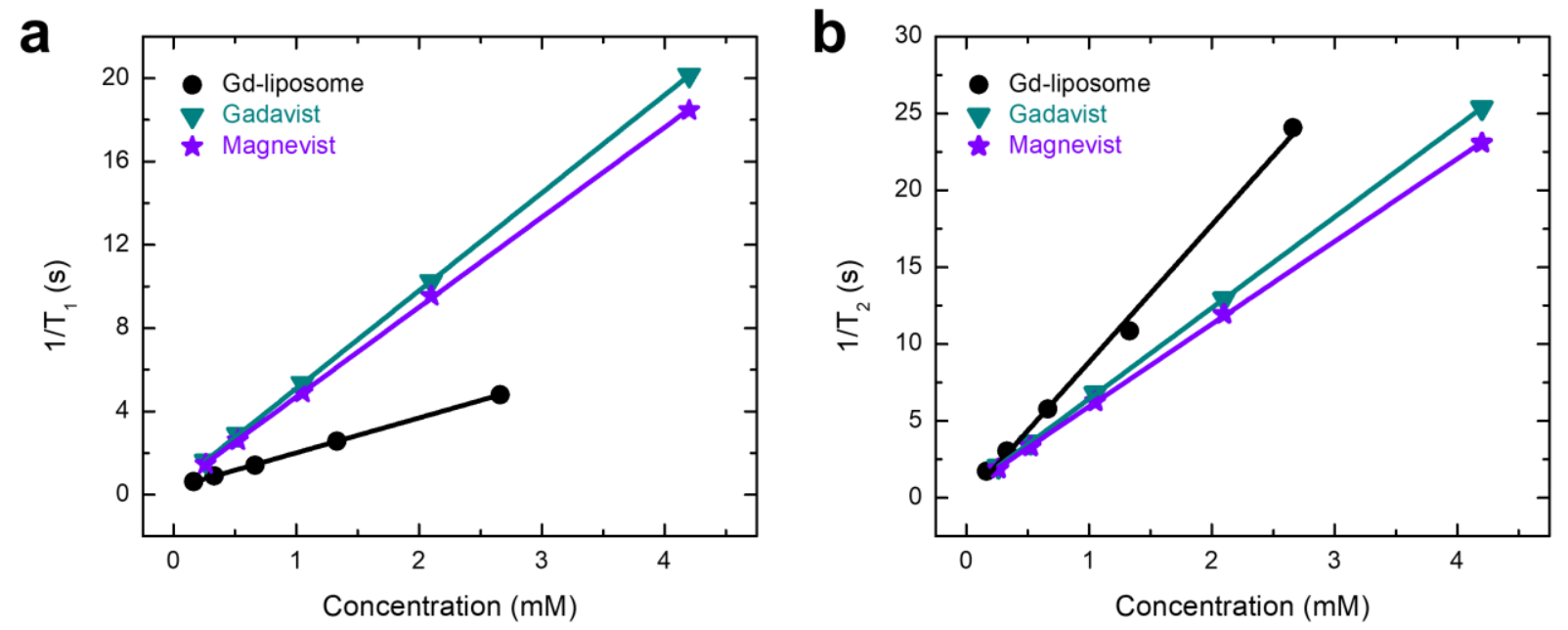

Figure 2. Relaxation rate measurement of paramagnetic liposomes (Gd-liposomes) and comparison with commercial contrast agents a) Longitudinal relaxation rate $\left(1 / T_{1}\right)$ measurement at different concentrations. Relaxivity $\left(\mathrm{r}_{1}\right)$ is calculated from the slopes of the fitted line; black represents paramagnetic liposome (Gd-liposome) with $\mathrm{r}_{1}=1.68 \mathrm{mM}^{-1} \mathrm{~s}^{-1}$, green represents Gadavist with $\mathrm{r}_{1}=4.70 \mathrm{mM}^{-1} \mathrm{~s}^{-1}$, purple represents Magnevist with $\mathrm{r}_{1}=4.32 \mathrm{mM}^{-1} \mathrm{~s}^{-}$ ${ }^{1}$ b) Transverse relaxation rate $\left(1 / \mathrm{T}_{2}\right)$ measurement at different concentration. Relaxivity $\left(\mathrm{r}_{2}\right)$ is calculated from the slopes of the fitted line; black represents paramagnetic liposome (Gdliposome) with $\mathrm{r}_{2}=8.93 \mathrm{~s}^{-1} \mathrm{mM}^{-1}$, green represents Gadavist with $\mathrm{r}_{2}=5.92 \mathrm{~s}^{-1} \mathrm{mM}^{-1}$, purple represents Magnevist with $\mathrm{r}_{2}=5.34 \mathrm{~s}^{-1} \mathrm{mM}^{-1}$ (Uncertainty in all the results are 10\%)

liposome or the outer water sphere. If the $\mathrm{Gd}^{3+}$ ions are in contact with the continuous water flow of the bulk water, then nanoparticles should behave like strong $T_{1}$ agents. However, our results are not consistent with such a conclusion. Therefore, we have hypothesized that $\mathrm{Gd}^{3+}$ ions might be preferentially located at the side of the inner aqueous core rather than the outer surface, restricting their access to the bulk water. Moreover, the high concentration of the $\mathrm{Gd}^{3+}$ in the small bilayer of 2-5 nm would act as a source of strong magnetic dipolar field, making the nanoparticles a good $T_{2}$ agent. In the following sections, we have compared MR images taken by standard $T_{2}$ and $T_{1}$ pulse sequences versus those with the active-feedback MR images obtained by employing a continuous wave pulse sequence. 


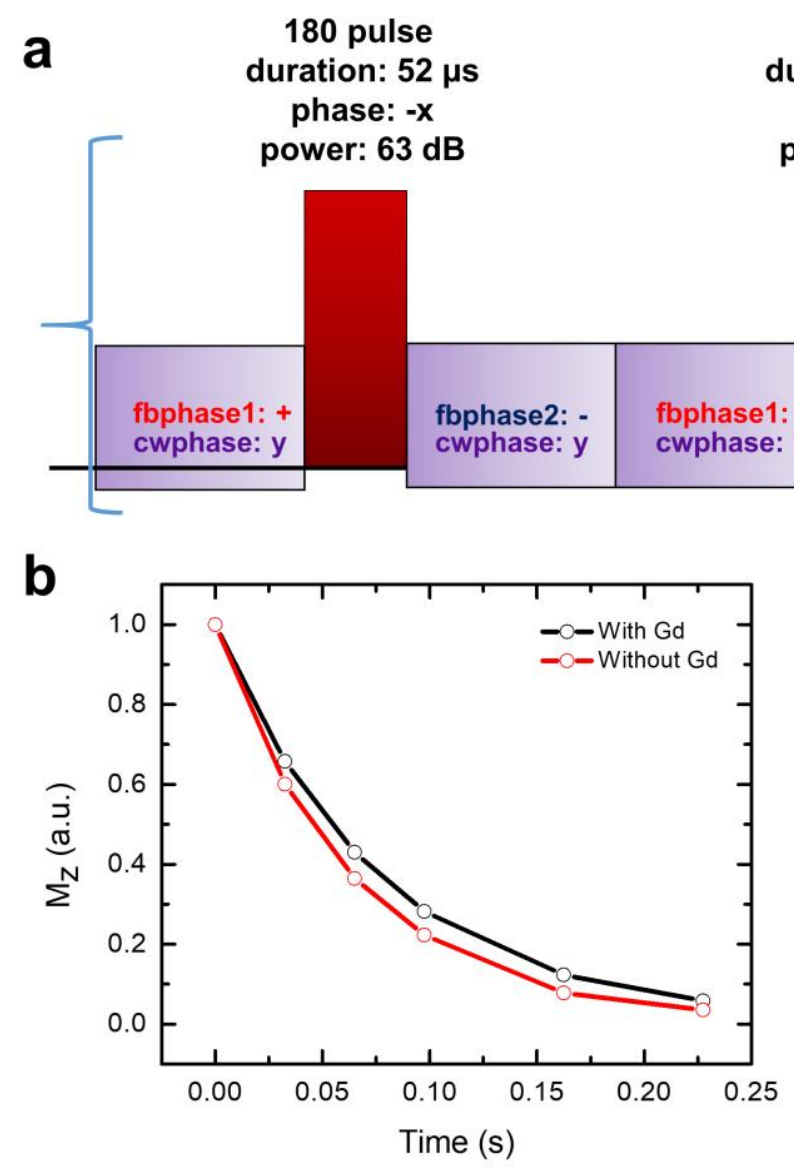


Additionally, phase alteration of the electronic feedback was done to enhance the natural radiation damping effect for contrast enhancement purposes under specific conditions, leading to potentially chaotic dynamics for simple-spin systems. ${ }^{29}$

Let $\mathbf{m}(\mathbf{r})=\mathbf{M}(\mathbf{r}) / \mathrm{Meq}$ be the normalized magnetization vector, where $\mathbf{M}(\mathbf{r})$ is magnetization vector and Meq is the equilibrium magnetization. The time evolution of $\mathbf{m}(\mathbf{r})$ is governed by the classical Bloch equations ${ }^{37,38}$ and depends: on the local magnetic field $\mathbf{B}(\mathbf{r}, \mathrm{t})$ defined in the rotating frame; the longitudinal $\left(\mathrm{T}_{1}\right)$ and transverse $\left(\mathrm{T}_{2}\right)$ relaxation times, and the self-diffusion coefficient (D). Information concerning the $\mathrm{CW}$ and active-feedback is contained in $\mathbf{B}(\mathbf{r}, \mathrm{t})$. In an appropriate frame of reference, we can write the local magnetic field $\mathbf{B}(\mathbf{r}, \mathrm{t})=\mathbf{B}_{\mathbf{z}}+\mathbf{B}_{\mathbf{c w}}$ $+\mathbf{B}_{+, \text {af }}$, where $\mathbf{B}_{\mathbf{z}}, \mathbf{B}_{\mathbf{c w}}, \mathbf{B}_{\mathbf{+ a f}}$ are the longitudinal component of the magnetic field, the contribution from $\mathrm{CW}$ and the contribution from active-feedback field, respectively. The active-feedback field can be modeled as a time-dependent magnetic field, ${ }^{39,40}$ given by

$\gamma \boldsymbol{B}_{+, a f}(t)=\frac{i e^{-i \varphi}}{\tau_{r} V} \iiint \boldsymbol{m}_{+}(\boldsymbol{r}, t) d^{3} \boldsymbol{r}$

Here, the active-feedback time constant $\tau_{\mathrm{r}}=\left(2 \pi \eta \mathrm{M}_{0} \mathrm{Q} \gamma\right)^{-1},{ }^{22} \eta$ and $\mathrm{Q}$ being the filling and quality factors of the coil, $\phi$, usually assumed to be zero, depends on the tuning efficiency of the probe, ${ }^{40}$ $\gamma$ is gyromagnetic ratio, $\mathbf{m}_{+}$is the transverse magnetization; and $\mathrm{V}$ is the total volume. The time evolution of the system will minimize $\int \boldsymbol{m}_{+}(\boldsymbol{r}) d^{3} \boldsymbol{r}$, and fixed points correspond to $\frac{\partial \boldsymbol{m}}{\partial t}=0$.

The time evolution of the system finally proceeds to the creation of stable fixed points (Figure S1). However, the effect of relaxation impedes the long-term stability of the fixed points, as the signal in vivo is not expected to last for more than a few hundreds of milliseconds. In fact, we would expect the in vivo signal to saturate completely long before the magnetization would be able to arrive at the stable fixed points. Despite the nature of the signal to eventually saturate, the 
simulations show that the evolution of the magnetization proceeds towards the fixed points. Considering a realistic system and modeling Gd-liposomes as sources of strong dipolar magnetic fields, the simulations results with the pulse sequence (Figure 3a) for the decay of the longitudinal magnetization $\left(\mathrm{M}_{\mathrm{z}}\right)$ with and without Gd-liposomes-are shown in Figure 3b. The figure shows that there is an en route difference of longitudinal decay between decays with and without Gdliposome. Assuming Gd-liposomes are targeted to the tumor site, this variation represents the difference between healthy and tumor tissue. Since, we are mostly interested in this change, pointto-point differences were calculated and Figure $3 \mathrm{c}$ shows the corresponding predicted temporal variation of $\mathrm{M}_{\mathrm{Z}}$ for Gd-liposomes. The predicted temporal variation exhibits biexponential behavior and provides a strong handle for discriminating between artifacts and spurious points.

\subsection{In vivo mice experiments}

In this experiment, the PEGylated nanoparticles were injected through the tail vein of a mouse at a concentration about $30 \mu \mathrm{g} / \mathrm{g}$, and we relied on passive targeting through the EPR effect. The in vivo mice experiments were done in a 7T Varian microimaging instrument. The MR images were taken before and after a few hours of injecting the Gd-liposomes in an otherwise identical experimental setup, where the position of the animal was kept fixed. Thus, any changes in the images taken before and after injecting Gd-liposome could be assigned to the presence of Gdliposomes in the mouse body. In order to image Gd-liposomes, we have acquired the direct differences of the intensity images taken before and after injecting Gd-liposome; these images will be referred to as difference images. The temporal evolution of the difference images has been obtained by taking such images at different time points. Figure 4 shows such a difference image 


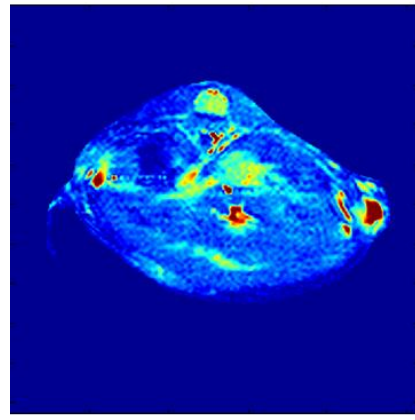

With Gd

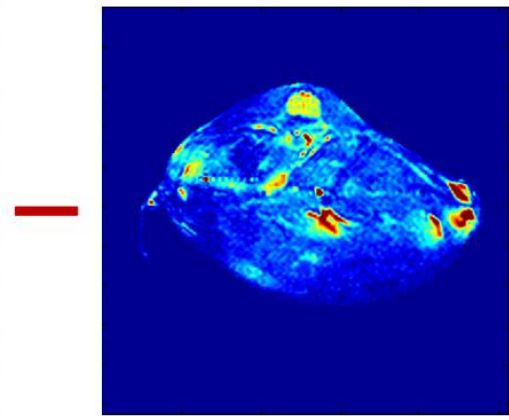

Without Gd

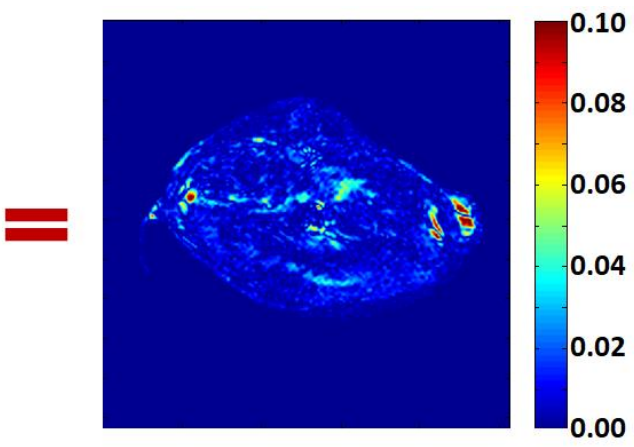

Difference image

Figure 4. Difference imaging: The absolute intensity difference between two images taken before and after injecting paramagnetic liposome (Gd-liposome), acquired by active-feedback pulse sequence for the same slice under identical experimental conditions.

taken with active-feedback technique (employing CW pulse sequence as shown in Figure 3a) for a part of the right hind leg of a mouse, where a tumor was grown. However, the difference image in Figure 4 lights up quite a few places in the image because of various artifacts making it difficult to accurately identify the position of the Gd-liposomes attached to the tumor. We have exploited an important characteristic feature of the active-feedback image with $\mathrm{CW}$ pulse sequence to eliminate artifacts and false imaging and compared with the images obtained by various conventional MR imaging protocols.

After injecting Gd-liposomes, the images were acquired under different preparation times of 33, 66, 99, 165 and $231 \mathrm{~ms}$ with our specially designed $\mathrm{CW}$ active-feedback pulse sequence. The difference images obtained at different preparation times are shown in Figure 5a. The longitudinal intensity differences correspond to the differences in longitudinal (z) magnetization and its temporal variation should be biexponential (Figure 3c) given by

$$
\text { Intensity Difference } \left.=A \times\left(e^{-\frac{t}{T_{A F 1}}}-e^{-\frac{t}{T_{A F 2}}}\right) \ldots \ldots \ldots \ldots \ldots \ldots \ldots \ldots . . . \ldots . . . . .2\right)
$$

where $\mathrm{T}_{\mathrm{AF} 2}$ and $\mathrm{T}_{\mathrm{AF} 1}$ are rise-time and decay-time constants respectively. A typical temporal variation of the difference images obtained from the experiment is shown in Figure $5 \mathrm{~b}$ along with 
(a)

Time(s) 0

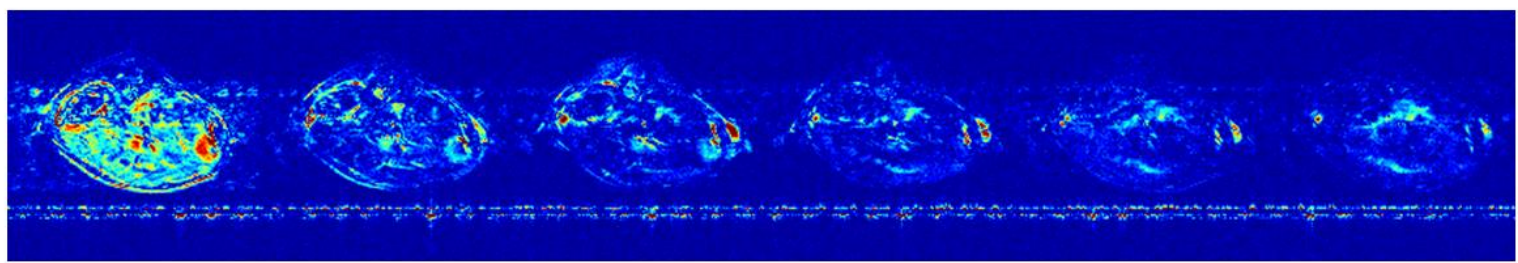

(b)

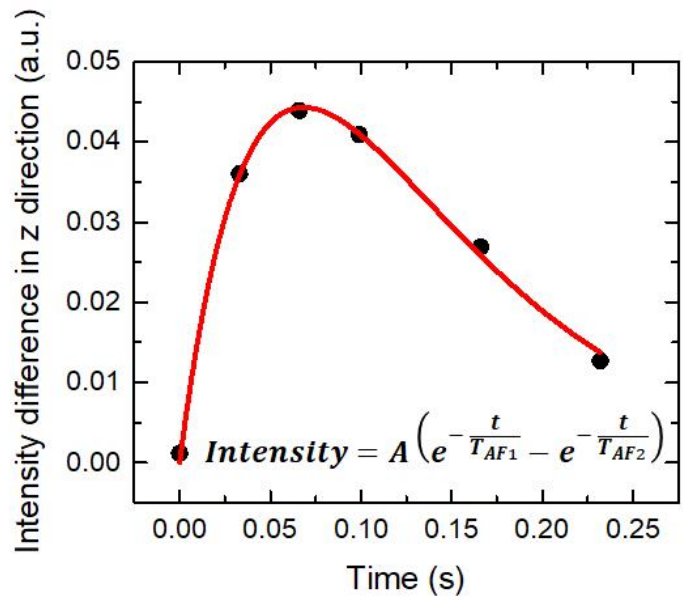

(c)

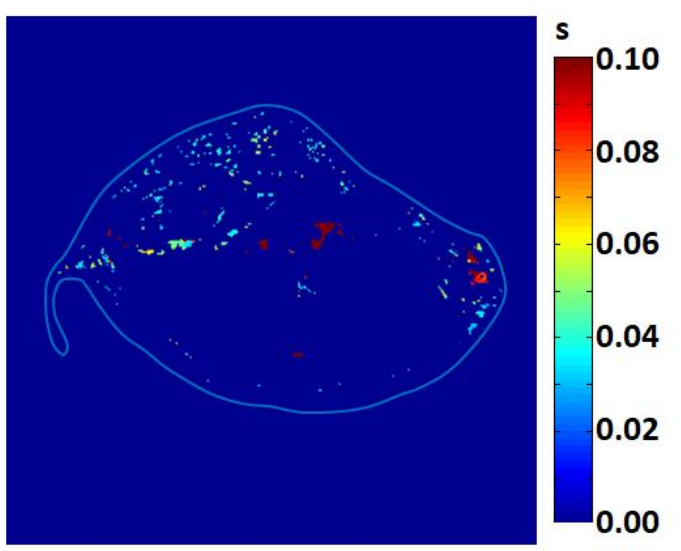

Figure 5. Time evolution of difference imaging a) The difference imaging is shown for different preparation times of 33, 66, 99, 165 and $231 \mathrm{~ms}$ for a particular slice. b) The temporal variation of the difference images fitted with a bi-exponential equation. c) Shows the parameter $\left(\mathrm{T}_{\mathrm{AF} 1}\right)$ at different points where goodness of fit $>0.7$, poorly fitted points have been removed as artifacts. The position of the tumor site is most intense and unambiguous.

a biexponential fit. The characteristic pattern of the plot in $5 b$ is in good accordance with the predicted temporal variation in Figure 3c. The intensity points whose temporal variation of longitudinal intensity difference cannot be fitted by a biexponential curve with the goodness of fit $\left(\mathrm{R}^{2}\right)>0.7$ were removed as artifacts or false imaging. The threshold $\mathrm{R}^{2}$ value has been chosen based on pixel-wise $\mathrm{R}^{2}$ mapping. In Figure $5 \mathrm{c}$, we show the values of the parameter $\mathrm{T}_{\mathrm{AF} 1}$ extracted from such fits at different points after eliminating artifacts.

In left panel of Figure 6, we show the difference images for the two mice and in the middle panel the same image for the points that follow the characteristic biexponential temporal variation with the goodness of fit $>0.7$ and eliminate the points that do not show this characteristic feature. 

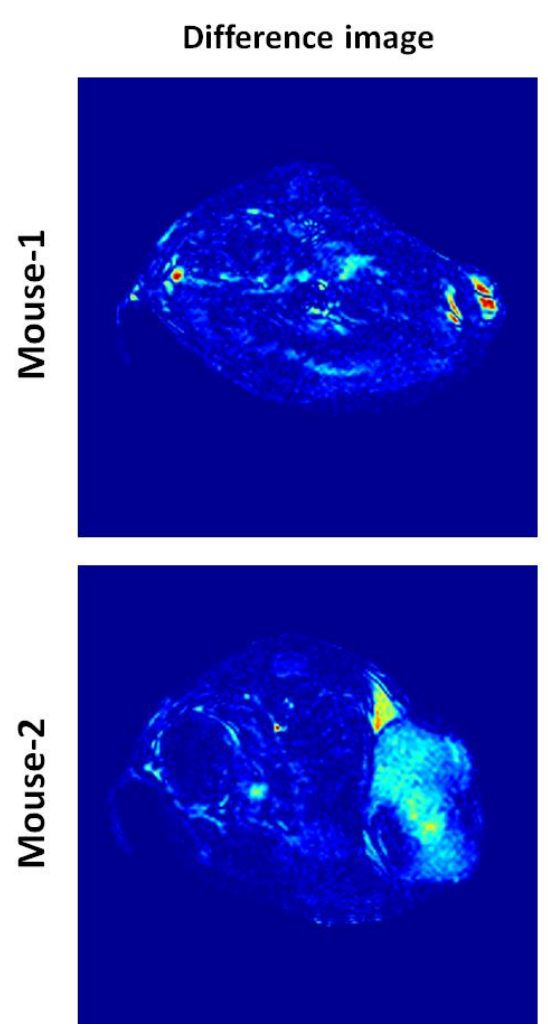

Parameter corrected

difference image

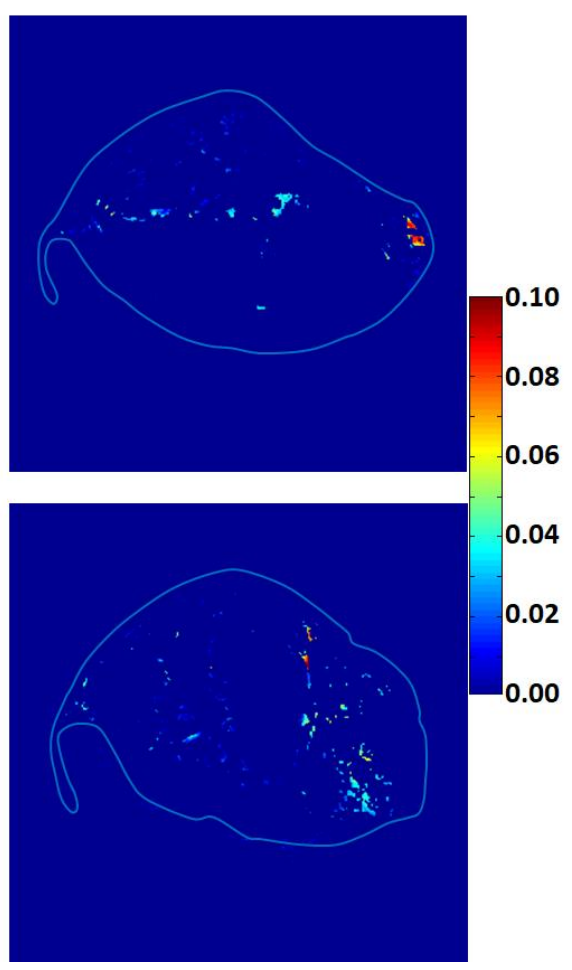

Overlaid image for

Gd position
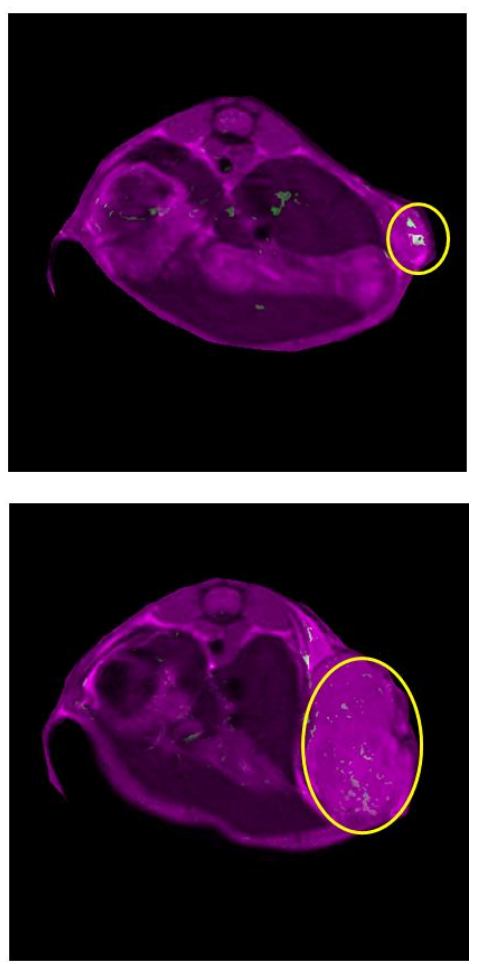

Figure 6. Parameter corrected difference image: Left column is showing the difference images for two mice. Middle column is demonstrating the same difference image for points that show the expected temporal behavior with the goodness of fit $>0.7$. The highest concentrations of paramagnetic liposomes (Gd-liposomes) are clearly seen at the tumor site. Right column is showing an overlay of this parameter corrected difference image with a standard MR image. See text for the details.

The middle panel shows the results for tumors in two mice (mouse-1 and mouse-2) with excellent contrast and without artifacts. Based on the observations and theoretical justifications, we claim that the images only contain the points where Gd-liposomes actually exist. The relative intensities are indicating the concentrations of the Gd-liposomes in this image. The right column of Figure 6 shows an overlay of the parameter-corrected difference images with a conventional MR image of the mouse to understand the anatomy. The yellow circle indicates the tumor position and the darker regions are parts of the liver. The images obtained by our new technique show clearly the positions 
of Gd-liposomes as white and grey dots with an excellent contrast, thus clearly displaying the location of the tumor.

This method was compared with conventional techniques such as inversion recovery $\mathrm{T}_{1}$, spin echo multi slice $\mathrm{T}_{2}$ (SEMS $\mathrm{T}_{2}$ ), gradient echo multi slice (GEMS $\mathrm{T}_{2}$ ) and $\mathrm{T}_{2}$-weighted techniques. We followed identical procedures of acquiring images before and after injecting the Gd-liposomes and obtained the parameter-fitted difference images for the conventional MR techniques to compare with our specially designed active feedback technique. In Figure 7, we show MRI images obtained by various conventional techniques versus our new technique for the two mice, clearly demonstrating that our new technique shows the best contrast to locate the Gdliposomes very efficiently without any background noise, artifacts or false imaging.

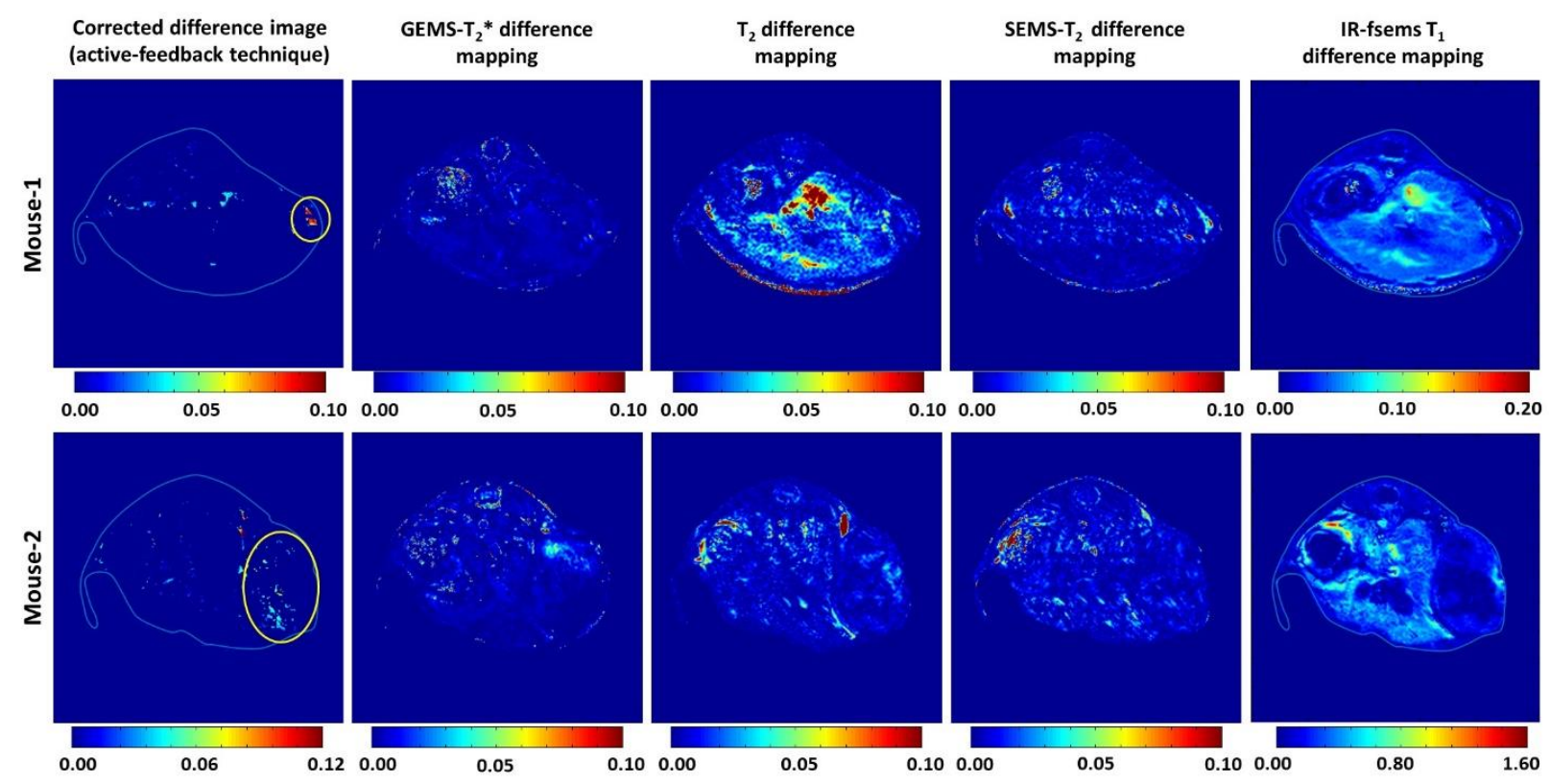

Figure 7. Comparison with conventional techniques: The comparison among different MR images taken using conventional techniques and our parameter-corrected difference imaging technique with active-feedback continuous-wave pulse sequence. Results are shown for mouse1 and mouse-2. The yellow circles show the positions of the tumor. See text for the details. 


\section{Gd $^{3+}-$ CONCENTRATION}

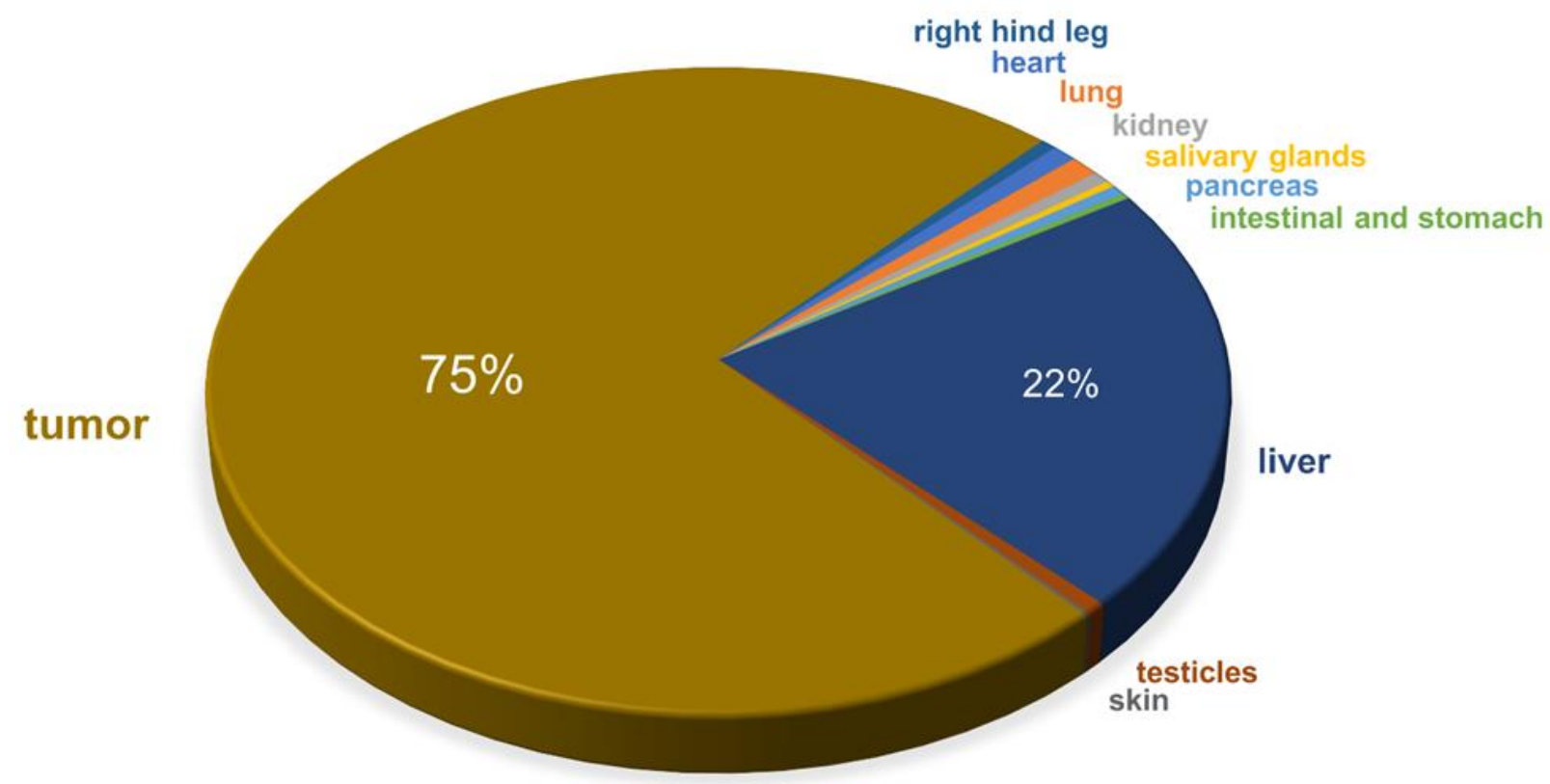

Figure 8: Inductively coupled mass spectrometry (ICP-MS) result: It demonstrates the concentration of $\mathrm{Gd}$ is $74.80 \mu \mathrm{g} / \mathrm{g}$ at tumor, $21.80 \mu \mathrm{g} / \mathrm{g}$ in the parts of the liver and $0.13-1.05$ $\mu \mathrm{g} / \mathrm{g}$ in other parts of the body. Uncertainties in the results are $10 \%$.

\subsection{Histopathology and targeting efficiency}

The mice were sacrificed after the experiment and histological sections were made to locate the position of the tumor shown in Figure 6 with yellow circles. Inductively coupled mass spectrometry (ICP-MS) results for Gd are displayed in Figure 8, showing the highest concentration of the $\mathrm{Gd}$ at the tumor site $(74.80 \mu \mathrm{g} / \mathrm{g})$ followed by a concentration of $21.8 \mu \mathrm{g} / \mathrm{g}$ at the parts of liver. Notably, the kidney has only $0.6 \mu \mathrm{g} / \mathrm{g}$ Gd, while other body parts have $0.13-1.05 \mu \mathrm{g} / \mathrm{g} \mathrm{Gd}$. In this context, it is worthwhile to mention that the clinical dosage for humans is usually $15 \mu \mathrm{g} / \mathrm{g}$ in regular use, however a 3 times higher dose of Magnevist and a 15 times higher dose for Gadavist were found to be well tolerated for healthy human. ${ }^{41,42}$ Figure 8 shows the percentages of Gd concentration in different parts of the mouse indicating $75 \%$ concentration at the tumor site, even for passive targeting. The use of active targeting ${ }^{6,43}$ could enhance this efficiency very significantly 
and better images could be obtained. Moreover, the incorporation of Gd-chelate into the bilayer of liposome should reduce the chances of leakage.

\section{Conclusions}

The ability to obtain artifact-free high-contrast images of the tumor site by encapsulating both the contrast agent and drug molecules in a nanoparticle plays a key role for the success of cancer theranostics. However, generally the encapsulation of the contrast agent reduces its ability to generate high-contrast images. In this work, we have demonstrated how high-contrast, artifact-free images could be obtained by employing a specially designed active feedback technique compared to available conventional MR imaging techniques. This newly developed MRI pulse sequence is based on an active-feedback technique employing a CW radio-frequency field in the transverse plane to extend the nonlinear evolution of the proton spins by preventing them from reaching equilibrium. The technique stabilizes and preserves the generated contrast in the context of fixedpoint dynamics. We have developed a novel analytical procedure to obtain high-contrast background-free and artifact-free images by exploiting the characteristic biexponential temporal variation of the intensity points of the difference images. The technique successfully lights up the positions of the nanoparticles accurately with a close correlation with histopathology and inductively-coupled mass-spectrometry results, where other conventional techniques give ambiguous images full of various artifacts.

Under clinical conditions, the field strength, filling and quality factors of the coil are usually not enough to produce an observable radiation damping field. We have shown how to overcome this problem with the implementation of an active feedback circuit, designed to read the 
free induction decay (FID), amplify and then retransmit the signal back onto the sample, thus emulating the effect of the radiation damping feedback field ${ }^{28}$ While the process is not the true radiation damping feedback field, the system exhibits the same non-linear response for the total magnetization and can thus be used in settings where radiation damping is negligible, i.e. at clinical setting using a low magnetic strength MRI.

The liposomal nanoparticles used in this work have been well studied for drug delivery and imaging purposes, however the sensitivity is poor because of insufficient interaction of $\mathrm{Gd}^{3+}$ with bulk water. ${ }^{13,16}$ This work shows a superior contrast mechanism to solve this long-standing problem. Here we have shown the nanoparticle accumulation at the tumor site through passive targeting. The dosage is within the clinical limit and could be reduced significantly by active targeting. This nanostructure provides high stability, limits the chances of releasing free Gd in the body, ensures better targeting to cancer cells and has the capability to be a potent theranostic for early cancer with high sensitivity without compromising contrast.

This protocol employing a characteristic temporal biexponential feature of the difference image under the new pulse sequence could be used as a powerful analytical tool for the robust detection of other paramagnetic nanostructures that generally suffer from limited contrast. Hence, our technique is an important step to achieve success in cancer theranostics using paramagnetic nanoparticles and it has the capability to generate high quality PET-like MR images.

\section{Methods}

\subsection{Preparation of Gd-liposomes}

A total of 112.5 micromole of 1,2-distearoyl-sn-glycero-3-phosphocholine(DSPC) (Corden pharma),1,2-distearoyl-sn-glycero-3-phosphoethanolamine-N-[amino(polyethylene- 
glycol)2000] (DSPE-PEG2000) (Corden pharma), DTPA-bis(stearylamide) (Gadolinium salt) (Gd-DTPA-BSA) ) (Avanti Polar Lipids, Inc), Cholestrol (Sigma Aldrich), (in a molar ratio of 1.10:0.15:0.75:1) were dissolved in chloroform/methanol (2:1) (Sigma Aldrich) mixture to yield a uniform solution of density typically between $10-20 \mathrm{mg} / \mathrm{ml} .{ }^{44,45}$ The homogeneous solution was evaporated under high vacuum at $40{ }^{\circ} \mathrm{C}$ and kept for 8 hours in a rotatory evaporator for the complete evaporation of organic solvent. The produced thin film was hydrated with phosphate buffer saline (10 mM PBS) (Sigma Aldrich) at $\mathrm{pH} 7.4$ for 1 hour above its transition temperature $\left(65^{\circ} \mathrm{C}\right)$ in a rotatory evaporator at a constant speed of about $60 \mathrm{rpm}$ with occasional vertexing and sonication. The resulting solution was passed through a 400 and $100 \mathrm{~nm}$ filters with 61 times each during extrusion using a mini extruder from Avanti Polar Lipid.

\subsection{Characterization of Gd-liposomes}

The size and morphology of the nanoparticles were verified with negatively stained transmission electron microscopy (TEM). The Gd-liposome solution was diluted 75-100 times and $6 \mu \mathrm{L}$ was taken on carbon coated copper grid, stained with $2 \%$ uranyl acetate solution, and dried. The grid was loaded with a Gatan specimen holder into T12 cryo-electron microscope (Electron Imaging Center for NanoMachines, California NanoSystems Institute) microscope for imaging. The images were acquired by operating the machine at $120 \mathrm{kV}$ with a Gatan $2 \mathrm{k} \times 2 \mathrm{k}$ CCD camera.

The hydrodynamic diameter was measured with dynamic light scattering (DLS) after diluting by 300 times in 10 mM PBS buffer using a Coulter Beckman Dynamic Light Scattering Analyzer - N4 Plus (UCLA Molecular Instrumentation Center).

\subsection{MRI measurements}

All MR images and relaxation measurements were performed in a $300 \mathrm{MHz}$, 7T Varian INOVA microimaging system with $5 \mathrm{~mm}$ bore. In order to obtain relaxation parameters, the 
nanoparticle solution was diluted to different concentrations and Gadavist (Radiologysolutions.Bayer) and Magnevist (Radiologysolutions.Bayer) were brought for comparison. Number of scans was taken as 1 and relaxation delay time was $3 \mathrm{~s}$ for each. $\mathrm{T}_{1}$ was measured using inversion recovery pulse sequence with inversion time (TI) ranging from $1 \mathrm{~ms}$ to 3 s. $\mathrm{T}_{2}$ was obtained with CPMG (Carr-Purcell-Meiboom-Gill) pulse sequence and the half of the interval between successive $180^{\circ}$ pulses in the CPMG pulse sequence $\left(\tau_{\mathrm{CP}}\right)$ was $=1 \mathrm{~ms}$. Time of echo (TE) was ranging from 4 to $300 \mathrm{~ms}$. The relaxation rates were plotted with the concentration of Gd to obtain a linear fit. The longitudinal and transverse relaxivity parameters $\left(r_{1}\right.$ and $\left.r_{2}\right)$ were calculated from the slope of the fitted straight line.

All MR images in this work were acquired after preparation stage by fast spin echo image sequence with repetition time $(\mathrm{TR})=7.5 \mathrm{~s}$, echo spacing time $=10 \mathrm{~ms}$, number of echos $=8$, number of scans $=1$, on transverse plane $($ axial $)$, the field of view $(\mathrm{FOV})=3.2 . \mathrm{cm} \times 3.2 \mathrm{~cm}$, thickness $=1 \mathrm{~mm}$, matrix size $=128 \times 128$, zero padding $=512 \times 512$. The $\mathrm{T}_{2}{ }^{*}$-weighted images were acquired by GEMS with flip angle $30^{\circ}, \mathrm{TR}=0.15 \mathrm{~s}$, TE is 5, 5.1, 6, 10, and $15 \mathrm{~ms}$ respectively. The $\mathrm{T}_{2}$-weighted images were acquired by SEMS sequence with $\mathrm{TR}=7.5 \mathrm{~s}$ and time of echo (TE or $\tau) 10,30,50$ and $70 \mathrm{~ms}$ respectively. $T_{1}$-weighted images were obtained by inversion recovery (IR) pulse sequence with TR=7.5 s and TI = $97 \mathrm{~ms}, 197 \mathrm{~ms}, 297 \mathrm{~ms}, 597 \mathrm{~ms}, 1.297 \mathrm{~s}$ and 3.097s. Active-feedback pulse sequence with $\mathrm{CW}$ was used, as shown in Figure 3 a) with different preparation times of $33 \mathrm{~ms}, 66 \mathrm{~ms}, 99 \mathrm{~ms}, 165 \mathrm{~ms}$ and $231 \mathrm{~ms}$. Details of hardware design of the active-feedback instrument could be found elsewhere. ${ }^{28,46}$

\subsection{Cell Culture}

The U87-MG cell lines were purchased from Bioresource Collection and Research Center (BCRC, Taiwan), derived from American Type Culture Collection (ATCC). It is cultured in 
minimum essential medium Eagle (MEM, Sigma-Aldrich) supplemented with $10 \%$ fetal bovine serum (FBS) (Gibco) and $100 \mathrm{U} / \mathrm{mL}$ penicillin-streptomycin antibiotics (Sigma-Aldrich). The cells were plated in T-75 flasks and maintained in a $5 \% \mathrm{CO}_{2}$ humidified incubator at $37^{\circ} \mathrm{C}$. About four flasks of cells were collected after washing with PBS three times and treatment with trypsin (Biowest) treatment. The suspended cells were centrifuged at $967.5 \mathrm{~g}$ for 5 minutes and concentrated to $1 \times 10^{5}$ cell $/ \mu \mathrm{L}$ before implantation.

\subsection{Animal Surgery}

All animal experiments were done in accordance with the regulations approved by the Institution Animal Care and Utilization Committee at National Taiwan University, Taiwan. The

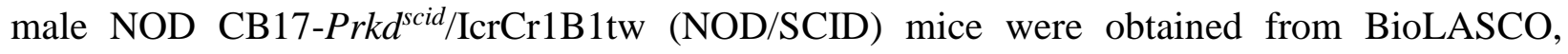
Taiwan. In vivo subcutaneous glioblastoma multiform (GBM) mouse models were prepared by injecting the U87-MG cells on the right hind legs of the mice. After injecting the mouse with the GBM cells, we waited for 8 days. Then, about 200 microliters of the nanoparticle solution with $2 \%$ heparin to prevent coagulation was injected (Hamilton 30 gauge syringe, equipped with pump to push the drug) through the tail of the mouse. During the measurement, mouse was anesthetized with about $1.5 \%$ isoflurane and its position was held fixed inside the instrument. The heart rate and respiratory signs of the mice were carefully monitored. The measurements were taken once just before injecting the Gd-liposomes nanoparticle solution and again a few hours after injecting the mouse with Gd-liposome solution. The mouse position was kept undisturbed between these two measurements. 


\subsection{Histopathology}

After the experiment, the mouse-1 was sacrificed, and its different tissues were dissected. Then all the dissected tissues were digested separately in concentrated nitric acid. Concentrated hydrofluoric acid was added as required to dissolve any undigested material. The solution was evaporated just to dryness and taken up in 2 washes of 5\% nitric acid for inductively coupled mass spectrometry (ICP-MS) analysis for elemental Gd at the Agilent 7500c quadrupole ICP-MS with hydrogen/helium octopole collision cell.

\subsection{Computer simulations for theoretical calculations}

Spin dynamics code was run using MATLAB (The Mathworks, Inc., South Natick, MA, USA). The Gd-nanoparticle was modeled as an impenetrable sphere of $168 \mathrm{~nm}$ diameter. The nanoparticle was placed at the center of a cubic box with volume fraction 0.000005 and the root mean square (rms) angular frequency shift at the particle surface $\Delta \omega_{\mathrm{r}}=50 \times 10^{7} \mathrm{rad} / \mathrm{s} .50000$ average voxels of magnetic moment with diffusing proton spins were simulated randomly. Constant background relaxation and inhomogeneity were modeled by taking a magnetic dipolar field at healthy tissue with $\Delta \omega_{\mathrm{r}}=40 \times 10^{7} \mathrm{rad} / \mathrm{s}$. The diffusion process was modeled as a random walk in a cubic lattice with each diffusion step of size $\sqrt{6 \times D \times t}$, where $\mathrm{D}$ is the diffusion coefficient of water $\left(\mathrm{D}=2.5 \times 10^{-5} \mathrm{~cm}^{2} / \mathrm{s}\right)$ and $\mathrm{t}$ is the diffusion time step. ${ }^{47,48}$ After each step, different magnetization offset and phase shift along the z-direction in the rotating frame experienced by the spins were calculated according to the following equation 4) for each voxel,

$B_{Z}(d, \theta)=\sqrt{\left(\frac{5}{4}\right)} r^{3} \Delta \omega_{r}\left(3 \cos ^{2} \theta-1\right) / \gamma d^{3}$

Here, $\gamma$ is the gyromagnetic ratio of proton, $r$ is the particle radius, $d$ is the distance from the particle center and $\theta$ is the angle between the $\mathrm{z}$-axis and the position where $\mathrm{B}_{\mathrm{z}}$ (magnetic field along $\mathrm{z}$ 
direction) is evaluated. Another set of 50000 average voxels of proton spins was taken to take care of healthy tissues, where no Gd-nanoparticle should be present, considering a perfect targeting case.

At every step the resonance offset at the rotating frame was evaluated according to the formula $\Delta \omega_{\text {offset }}=\gamma B_{z}$ for each spin. The average transverse magnetizations experienced by both sets of spins were numerically integrated taking care of $\mathrm{CW}$ pulse and accordingly substituted for

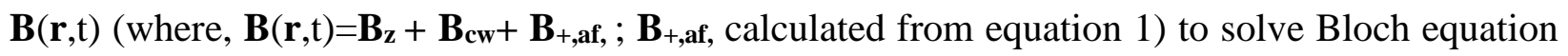
for every voxel.

\section{Acknowledgements}

We gratefully acknowledge Prof. Ying-Chih Lin, Prof. William Gelbart, Prof. Charles M.

Knobler, Prof. Neil K. Garg for lab facilities, Dr. Shane Quee Hee (ICP-MS facility at School of Public Health, UCLA) for ICP-MS measurements, National Taiwan University for in vivo facility and NSF-EAPSI for travel expenses to commute Taiwan. This work was supported by NSF grant CHE-1112574 and CHE-1416598, University of California Cancer Research Award (CRR-13201412), and the Hirshberg Foundation for Pancreatic Cancer Research.

\section{Competing Interests}

The authors declare that there is no competing interest. 


\section{References}

(1) Singh, R.; Lillard, J. W. Nanoparticle-Based Targeted Drug Delivery. Exp. Mol. Pathol. 2009, 86 (3), 215-223. https://doi.org/10.1016/J.YEXMP.2008.12.004.

(2) Sunderland, C. J.; Steiert, M.; Talmadge, J. E.; Derfus, A. M.; Barry, S. E. Targeted Nanoparticles for Detecting and Treating Cancer. Drug Dev. Res. 2006, 67 (1), 70-93. https://doi.org/10.1002/ddr.20069.

(3) Kelkar, S. S.; Reineke, T. M. Theranostics: Combining Imaging and Therapy. Bioconjug. Chem. 2011, 22 (10), 1879-1903. https://doi.org/10.1021/bc200151q.

(4) Janib, S. M.; Moses, A. S.; MacKay, J. A. Imaging and Drug Delivery Using Theranostic Nanoparticles. Adv. Drug Deliv. Rev. 2010, 62 (11), 1052-1063.

https://doi.org/10.1016/j.addr.2010.08.004.

(5) Allen, T. M.; Cullis, P. R. Liposomal Drug Delivery Systems: From Concept to Clinical Applications. Adv. Drug Deliv. Rev. 2013, 65 (1), 36-48.

https://doi.org/10.1016/J.ADDR.2012.09.037.

(6) Hosta-Rigau, L.; Schattling, P.; Teo, B. M.; Lynge, M. E.; Städler, B. Recent Progress of Liposomes in Nanomedicine. J. Mater. Chem. B 2014, 2 (39), 6686-6691. https://doi.org/10.1039/C4TB00825A.

(7) Mikhaylov, G.; Mikac, U.; Magaeva, A. A.; Itin, V. I.; Naiden, E. P.; Psakhye, I.; Babes, L.; Reinheckel, T.; Peters, C.; Zeiser, R.; et al. Ferri-Liposomes as an MRI-Visible DrugDelivery System for Targeting Tumours and Their Microenvironment. Nat. Nanotechnol. 2011, 6 (9), 594-602. https://doi.org/10.1038/nnano.2011.112.

(8) Shin, T.; Choi, J.; Yun, S.; Kim, I.; Song, H.; Kim, Y. T 1 and T 2 Dual-Mode MRI Contrast Agent for Enhancing Accuracy by Engineered Nanomaterials. ACS Nano 2014, 8 (4), 3393-3401. https://doi.org/10.1021/nn405977t.

(9) Delanian, S.; Baillet, F.; Huart, J.; Lefaix, J.-L.; Maulard, C.; Housset, M. Successful Treatment of Radiation-Induced Fibrosis Using Liposomal CuZn Superoxide Dismutase: Clinical Trial. Radiother. Oncol. 1994, 32 (1), 12-20. https://doi.org/10.1016/0167- 
8140(94)90444-8.

(10) Unezaki, S.; Maruyama, K.; Takahashi, N.; Koyama, M.; Yuda, T.; Suginaka, A.; Iwatsuru, M. Enhanced Delivery and Antitumor Activity of Doxorubicin Using LongCirculating Thermosensitive Liposomes Containing Amphipathic Polyethylene Glycol in Combination with Local Hyperthermia. Pharm. Res. 1994, 11 (8), 1180-1185. https://doi.org/10.1023/A:1018949218380.

(11) Northfelt, D. W.; Dezube, B. J.; Thommes, J. A.; Miller, B. J.; Fischl, M. A.; FriedmanKien, A.; Kaplan, L. D.; Du Mond, C.; Mamelok, R. D.; Henry, D. H. PegylatedLiposomal Doxorubicin versus Doxorubicin, Bleomycin, and Vincristine in the Treatment of AIDS-Related Kaposi's Sarcoma: Results of a Randomized Phase III Clinical Trial. J. Clin. Oncol. 1998, 16 (7), 2445-2451. https://doi.org/10.1200/JCO.1998.16.7.2445.

(12) Alberts, D. S.; Muggia, F. M.; Carmichael, J.; Winer, E. P.; Jahanzeb, M.; Venook, A. P.; Skubitz, K. M.; Rivera, E.; Sparano, J. A.; Dibella, N. J.; et al. Efficacy and Safety of Liposomal Anthracyclines in Phase I/II Clinical Trials. Semin. Oncol. 2004, 31, 53-90. https://doi.org/10.1053/J.SEMINONCOL.2004.08.010.

(13) Kamaly, N.; Miller, A. D. Paramagnetic Liposome Nanoparticles for Cellular and Tumour Imaging. Int. J. Mol. Sci. 2010, 11 (4), 1759-1776. https://doi.org/10.3390/ijms11041759.

(14) Strijkers, G. J.; Mulder, W. J. M.; Van Heeswijk, R. B.; Frederik, P. M.; Bomans, P.; Magusin, P. C. M. M.; Nicolay, K. Relaxivity of Liposomal Paramagnetic MRI Contrast Agents. Magn. Reson. Mater. Physics, Biol. Med. 2005, 18 (4), 186-192. https://doi.org/10.1007/s10334-005-0111-y.

(15) Unger, E.; Shen, D. K.; Wu, G.; Fritz, T. Liposomes as MR Contrast Agents: Pros and Cons. Magn. Reson. Med. 1991, 22 (2), 304-308. https://doi.org/10.1002/mrm.1910220229.

(16) Tilcock, C.; Unger, E.; Cullis, P.; Macdougall, P. Liposomal Gd-DTPA: Preparation and Characterization of Relxavity. Radiology 1989, 171, 77-80.

(17) Hak, S.; Sanders, H. M. H. F.; Agrawal, P.; Langereis, S.; Grüll, H.; Keizer, H. M.; Arena, F.; Terreno, E.; Strijkers, G. J.; Nicolay, K. A High Relaxivity Gd(III)DOTA-DSPE- 
Based Liposomal Contrast Agent for Magnetic Resonance Imaging. Eur. J. Pharm.

Biopharm. 2009, 72 (2), 397-404. https://doi.org/10.1016/j.ejpb.2008.09.017.

(18) Laurent, S.; Vander Elst, L.; Thirifays, C.; Muller, R. N. Relaxivities of Paramagnetic Liposomes: On the Importance of the Chain Type and the Length of the Amphiphilic Complex. Eur. Biophys. J. 2008, 37 (6), 1007-1014. https://doi.org/10.1007/s00249-0080331-y.

(19) Liang, Z.-P.; Lauterbur, P. C.; IEEE Engineering in Medicine and Biology Society. Principles of Magnetic Resonance Imaging : A Signal Processing Perspective; SPIE Optical Engineering Press, 2000.

(20) Zimmerman, R. A.; Gibby, W. A.; Carmody, R. F. Neuroimaging : Clinical and Physical Principles; Springer New York, 2000.

(21) Haacke, E. M.; Xu, Y.; Cheng, Y.-C. N.; Reichenbach, J. R. Susceptibility Weighted Imaging (SWI). Magn. Reson. Med. 2004, 52 (3), 612-618. https://doi.org/10.1002/mrm.20198.

(22) Bloembergen, N.; Pound, R. V. Radiation Damping in Magnetic Resonance Experiments. Phys. Rev. 1954, 95 (1), 8-12. https://doi.org/10.1103/PhysRev.95.8.

(23) Lin, Y. Y.; Lisitza, N.; Ahn, S.; Warren, W. S. Resurrection of Crushed Magnetization and Chaotic Dynamics in Solution NMR Spectroscopy. Science 2000, 290 (5489), 118122.

(24) Datta, S.; Huang, S. Y.; Lin, Y. Y. Contrast Enhancement by Feedback Fields in Magnetic Resonance Imaging. J. Phys. Chem. B 2006, 110 (44), 22071-22078. https://doi.org/10.1021/jp060658k.

(25) Huang, S. Y.; Furuyama, J. K.; Lin, Y.-Y. Designing Feedback-Based Contrast Enhancement for in Vivo Imaging. Magn. Reson. Mater. Physics, Biol. Med. 2007, 19 (6), 333-346. https://doi.org/10.1007/s10334-006-0061-z.

(26) Huang, S. Y.; Wolahan, S. M.; Mathern, G. W.; Chute, D. J.; Akhtari, M.; Nguyen, S. T.; Huynh, M. N.; Salamon, N.; Lin, Y.-Y. Improving MRI Differentiation of Gray and White Matter in Epileptogenic Lesions Based on Nonlinear Feedback. Magn. Reson. Med. 2006, 
56 (4), 776-786. https://doi.org/10.1002/mrm.20987.

(27) Li, Z.; Hsu, C.-H.; Dimitrov, N.; Hwang, D. W.; Chang, H.-W.; Hwang, L.-P.; Lin, Y.-Y. Sensitive Imaging of Magnetic Nanoparticles for Cancer Detection by Active Feedback MR. Magn. Reson. Med. 2015, 74 (1), 33-41. https://doi.org/10.1002/mrm.25632.

(28) Chen, Y.-W.; Hsu, C.-H.; Hwang, D. W. Novel MRI Contrast Development by Lock-in Suppression. Magn. Reson. Med. 2014, 71 (5), 1676-1681.

https://doi.org/10.1002/mrm.25162.

(29) Abergel, D. Chaotic Solutions of the Feedback Driven Bloch Equations. Phys. Lett. A 2002, 302 (1), 17-22. https://doi.org/10.1016/S0375-9601(02)01079-4.

(30) Louis-Joseph, A.; Abergel, D.; Lallemand, J.-Y. Neutralization of Radiation Damping by Selective Feedback on a 400 MHz NMR Spectrometer. J. Biomol. NMR 1995, 5 (2), 212 216. https://doi.org/10.1007/BF00208813.

(31) Broekaert, P.; Jeener, J. Suppression of Radiation Damping in NMR in Liquids by Active Electronic Feedback. J. Magn. Reson. Ser. A 1995, 113 (1), 60-64. https://doi.org/10.1006/JMRA.1995.1056.

(32) Abergel, D.; Louis-Joseph, A.; Lallemand, J. Y. Amplification of Radiation Damping in a 600-MHz NMR Spectrometer: Application to the Study of Water-Protein Interactions. J. Biomol. NMR 1996, 8 (1), 15-22. https://doi.org/10.1007/BF00198136.

(33) Abergel, D.; Carlotti, C.; Louis-Joseph, A.; Lallemand, J. Improvements in RadiationDamping Control in High-Resolution NMR. J. Magn Reson Ser B 1995, 109, 218-222.

(34) Abergel, D.; Louis-Joseph, A.; Lallemand, J.-Y. Self-Sustained Maser Oscillations of a Large Magnetization Driven by a Radiation Damping-Based Electronic Feedback. $J$. Chem. Phys. 2002, 116 (16), 7073-7080. https://doi.org/10.1063/1.1462583.

(35) Hobson, R. F.; Kaiser, R. Some Effects of Radiation Feedback in High Resolution NMR. J. Magn. Reson. 1975, 20 (3), 458-474. https://doi.org/10.1016/0022-2364(75)90003-7.

(36) Louis-Joseph, A.; Abergel, D.; Lebars, I.; Lallemand, J. Y. Enhancement of Water Suppression by Radiation Damping-Based Manipulation of Residual Water in Jump and 
Return NMR Experiments. Chem. Phys. Lett. 2001, 337 (1-3), 92-96.

https://doi.org/10.1016/S0009-2614(01)00174-9.

(37) Bloch, F. Nuclear Induction. Phys. Rev. 1946, 70 (7-8), 460-474.

https://doi.org/10.1103/PhysRev.70.460.

(38) Abragam, A. The Principles of Nuclear Magnetism; Clarendon Press, 1983.

(39) Vlassenbroek, A.; Jeener, J.; Broekaert, P. Radiation Damping in High Resolution Liquid NMR: A Simulation Study. J. Chem. Phys. 1995, 103 (14), 5886-5897. https://doi.org/10.1063/1.470468.

(40) Huang, S. Y.; Anklin, C.; Walls, J. D.; Lin, Y. Y. Sizable Concentration-Dependent Frequency Shifts in Solution NMR Using Sensitive Probes. J. Am. Chem. Soc. 2004, 126 (49), 15936-15937. https://doi.org/10.1021/ja046208s.

(41) Bayer in Radiology $\mid$ Gadavist ${ }^{\circledR}$ (gadobutrol) injection $1 \mathrm{mmol} / \mathrm{mL}$ https://www.radiologysolutions.bayer.com/products/mr/contrast/gadavist/ (accessed Nov 18, 2018).

(42) Bayer in Radiology $\mid$ Magnevist® (gadopentetate dimeglumine) injection $0.5 \mathrm{mmol} / \mathrm{mL}$ https://www.radiologysolutions.bayer.com/products/mr/contrast/magnevist/ (accessed Nov 18, 2018).

(43) Takeda, K.; Kobari, M.; Akaishi, S.; Matsuno, S. Targeting Pancreatic Chemotherapy Cancer Using Liposome against Antibody-Combined. J. Exp. Med. 1994, 175, 29-42.

(44) Mulder, W. J. M.; Strijkers, G. J.; Griffioen, A. W.; Bloois, L. van; Molema, G.; Storm, G.; Koning, G. A.; Nicolay, K. A Liposomal System for Contrast-Enhanced Magnetic Resonance Imaging of Molecular Targets. Bioconjugate Chem. 2004, 15, 799-806. https://doi.org/10.1021/bc049949r.

(45) Van Tilborg, G. A. F.; Mulder, W. J. M.; Deckers, N.; Storm, G.; Reutelingsperger, C. P. M.; Strijkers, G. J.; Nicolay, K. Annexin A5-Functionalized Bimodal Lipid-Based Contrast Agents for the Detection of Apoptosis. Bioconjug. Chem. 2006, 17 (3), 741-749. https://doi.org/10.1021/bc0600259. 
(46) Hsu, C.-H. Early Cancer Detection by Active Feedback Magnetic Resonance Molecular Imaging, National Tsing-Hua University, 2014.

(47) Gillis, P.; Moiny, F.; Brooks, R. A. OnT2-Shortening by Strongly Magnetized Spheres: A Partial Refocusing Model. Magn. Reson. Med. 2002, 47 (2), 257-263. https://doi.org/10.1002/mrm.10059.

(48) Matsumoto, Y.; Jasanoff, A. T2 Relaxation Induced by Clusters of Superparamagnetic Nanoparticles: Monte Carlo Simulations. Magn. Reson. Imaging 2008, 26 (7), 994-998. https://doi.org/10.1016/J.MRI.2008.01.039. 